\title{
Metadichol ®. A Novel Inverse Agonist of Aryl Hydrocarbon Receptor (AHR) and NRF2 Inhibitor
}

\section{Raghavan PR*}

Nanorx Inc., Chappaqua, New York, USA

\begin{abstract}
Metadichol ${ }^{\circledR}$ a novel nano emulsion of lipid alcohols is an Inverse agonist of Vitamin D Receptor (VDR). In this communication, we show that Metadichol is an inverse agonist of the nuclear receptor AHR (Aryl Hydrocarbon receptor) and also an inhibitor of NRF2 (Nuclear factor (erythroid-derived 2)-like 2) and is a transcription factor that is ubiquitously expressed at low levels in all human organs and regulates a primary cellular defense mechanism, tight regulation to maintain cellular homeostasis. AHR is highly expressed in a broad panel of tumors, AHR is induced by 2,3,7,8-tetrachloride-benzo-p-dioxin (TCDD) Metadichol ${ }^{\circledR}$ as a inverse agonist against AHR could be potentially useful in the treatment of such diseases. Strong in vivo evidence suggests that TCDD can stimulate cross-talk between AHR and Nrf2. The constitutive up regulation of Nrf2 signaling appears to drive the cellular proliferation and resistance to chemotherapy in various cancers. Therefore, pharmacological inhibition of Nrf2 by Metadichol $®$ holds promise as a therapeutic strategy in chemo resistant forms of cancer.
\end{abstract}

Keywords: Metadichol; Nano lipid; Long chain saturated alcohols; VDR; Inverse agonist; Protean agonist; AHR; Aryl Hydrocarbon Receptor; Inverse agonist; Antagonist; Chemo resistant cancers; Proliferation; Metastasis; Constitutive activity; NRF2

\section{Introduction}

Metadichol $^{\circledR}$ is a nano formulation of long chain alcohols that is an inverse agonist of Vitamin D receptor [1]. AHR (Aryl hydrocarbon receptor) like VDR a ligand activated transcription factor. It forms a nuclear hetero dimer with the AHR nuclear trans locator (ARNT) protein to activate gene expression. AHR [2] is known to mediate most of the toxic and carcinogenic effects of a wide variety of environmental contaminants such as dioxin TCDD (2,3,7,8-tetrachlorodibenzo[p]-dioxin). Many dietary compounds and products of commensal flora activate AHR signaling at physiologically relevant doses and with significant potency $[3,4]$. This allows targeting AHR to treat inflammatory and autoimmune diseases [5]. It is conceivable therefore that inhibition of AHR activity by antagonists could result in beneficial anti-inflammatory actions. Evidence for such anti-inflammatory effects has recently been identified by use of the AHR antagonist $\mathrm{CH}-223191$ [6] which represses TH17. Another high affinity AHR antagonist, Stemregenin 1 (SR1) has been shown to increase the proliferation of human hematopoietic stem cells in vitro [7]. AHR provides a molecular pathway through which environmental factors modulate the immune response and, consequently, the development of immune-mediated disorders. Agonists of the AHR receptor play an integral role in T-cell function, promoting a TH2/TH1 switch resulting in a TH1 bias [8]. AHR is usually present in epithelial cells where it is activated by different ligands. Elevated levels of AHR are seen in breast and other cancers where AHR is always activated by depressing anti-tumor immune responses. AHR up regulation allows tumor cells to migrate, invade local tissue and metastasize to other organs. AHR is highly expressed in a wide variety of tumors. Molecules with antagonistic AHR activity could be considered potential candidates for the treatment of such diseases. AHR is widely expressed in Pancreatic [9], Prostate [10], Urinary tract [11] Lung [12] Esophagus [13], Pituitary [14], gliomas [15] For long-term survival of cancers treatments activating the patient's own immune system is an important approach. For a successful treatment, other aspects of cancer progression, like cancer stem cells, metastatic behavior and decreased tumor cell apoptosis need to be targeted simultaneously. AHR also plays a role in producing "cancer stem cells", that are resistant to chemotherapies and this leads to lethal relapse and metastasis even years after "successful" chemo- or radiotherapy. Inhibitors not only boost anti-tumor immunity, but they also suppress metastasis and the formation of chemo-resistant cancer stem cells. Inhibiting AHR activity holds the promise of a non-toxic therapy that targets crucial tumor progressing processes at the same time. AHR antagonists can have therapeutic immunological value [16]

\section{Nrf2}

The Nuclear factor erythroid 2-related factor 2 (Nrf2) is a transcription factor regulating a variety of genes for antioxidant and detoxification enzymes such as glutathione S-transferase A2 (GSTA2) and NADPH quinone oxido-reductase 1 (NQO1) in response to oxidative and xenobiotic stress [17]. Antioxidants counteract oxidative stress, employing a variety of techniques to diminish ROS levels in the intracellular environment. Free radicals are elevated during cancer and activate signaling pathways of cell proliferation and migration, and can cause DNA damage leading to mutations. Nrf2 signaling pathway consists of three main components: (a) Keap1, (b) Nrf2, and (c) the Antioxidant response element (ARE). These three elements function respectively as the sensor, controller, and responder [18]. Constitutive activation of Nrf2 occurs in many cancers. Aberrant activation of Nrf2 is correlated to tumor progression, chemo resistance, and radiation resistance. NRF2 activations are regarded as beneficial, but recent work suggests otherwise [19]. Increased NRF2 activity from mutations

*Corresponding author: PR Raghavan, Founder and CEO, Nanorx Inc. PO Box 131, Chappaqua, New York, 10514, USA, Tel: 9146710224; E-mail: raghavan@nanorxinc.com

Received August 16, 2017; Accepted September 25, 2017; Published September 26, 2017

Citation: Raghavan PR (2017) Metadichol ®. A Novel Inverse Agonist of Ary Hydrocarbon Receptor (AHR) and NRF2 Inhibitor. J Cancer Sci Ther 9: 661-668. doi:10.4172/1948-5956.1000489

Copyright: @ 2017 Raghavan PR. This is an open-access article distributed unde the terms of the Creative Commons Attribution License, which permits unrestricted use, distribution, and reproduction in any medium, provided the original author and source are credited. 
in NFE2L2 or KEAP1 is contributes to chemotherapy resistance. Excessive induction of NRF2 genes in cancer cells, and therefore superior protection against cell stress may confer a survival advantage [20].

NRF2 is being researched as a drug target in many diseases like COPD and Multiple sclerosis and as inhibitors in enhancing cancer cell susceptibility to chemotherapy. Sulforaphane is useful in COPD, rescuing impaired phagocytosis in alveolar macrophages and breaking down macrophage resistance to gluco corticosteroid treatment via HDAC2 denitrosylation [21,22]. Dimethyl fumarate has shown promise in clinical multiple sclerosis trials, in a mouse MS model (experimental autoimmune encephalomyelitis, or EAE). It attenuated some of the damaging effects of the disease in dependence on Nrf2. Treatment enhanced Nrf2 stabilization and activity [23]. Ren et al. [24] studied the effect of Brusatol a compound found in a plant extract that inhibits the NRF2 pathway. In lung cancer cells Brusatol increases ubiquitination of NRF2 leaving other signaling pathways unaffected, and renders both cancer cell lines and xenografts susceptible to the chemotherapeutic agents like Cisplatin.

Nrf2 is essential to maintaining the cellular redox homeostasis, providing protection against oxidative or electrophilic stresses, and suppressing tumor promotion and progression [25]. In vivo, Nrf2knockout mice show greater damage in organs such as the lung, liver, and kidney when exposed to chemical toxins such as cigarette smoke or hypoxia [26]. Additionally, Nrf2-knockout mice are more prone to developing diseases related to oxidative stress and cancers [27]. Constitutive elevation of Nrf2 expression and Nrf2-ARE activity occurs in many cancers and is involved in their chemo-resistance and radiation resistance. Ideally, inhibitors should block the Nrf2-ARE signaling pathway in cancer cells, while leaving healthy cells unaffected. Although expression of $\mathrm{Nrf} 2$ can be suppressed experimentally by gene therapy, implementing them clinically in cancer treatment is a major hurdle. It is important to identify small-molecular Nrf2 inhibitors that block Nrf2 signaling pathways specifically in cancer cells. Thus, there is a need for a molecule that is specific and has bioactivity and also has no toxicity. Metadichol ${ }^{\circledR}$ as we shall show may fit that need.

\section{NRF2 and AHR}

Both AHR and NRF2 signaling regulate the expression of genes affecting the metabolism of xenobiotics. Miao et al. [28] showed that Nrf2 gene transcription is modulated by AHR activation. NRF2 also directly modulates AHR signaling, highlighting the dependent nature of interactions between these two pathways [29]. NRF2 controls AHR signaling to expand its function of NRF2 to include the metabolism to include xenobiotics and carcinogens via CYPs and adipogenesis. AHR and Nrf2 double knockout mice studies offer additional evidence for interactions between these pathways [30]. Cooperation between AHR and Nrf2 has biological implications. AHR has to do with carcinogenesis, whereas Nrf2 is involved with cytoprotection. The challenge is to exploit this to a to therapeutic advantage and to improve chemo preventive strategies.

\section{Experimental}

AHR nuclear receptor assay was outsourced and performed by Indigibio sciences PA, USA.

Reporter cells were treated with seven concentrations of respective test compounds, starting at $10 \mathrm{mg} / \mathrm{ml}$ and following with six more 3.33-fold dilutions. All treatment concentrations were performed in triplicate. Reporter cells were also treated with vehicle only as a control. Performance of the human NR agonist and antagonist assays were validated by performing a Reference Agonist (MeBIOP), and Antagonist (GNF 351) dose response curves

\section{Assay methods}

Plasmids. This study utilized proprietary nuclear receptor reporter cells expressing either the full-length nuclear receptor sequence (AHR,) or a hybrid receptor comprising the N-terminal Gal4 DNA binding domain fused to the ligand binding domain of the specific human nuclear receptor. The reporter vectors used in these studies comprise the firefly luciferase gene functionally linked to either an upstream NR response element (NRE) or the Gal4 activation sequence (UAS).

\section{Compound handling}

Test compound was delivered as solution dissolved in water. Stock of test compounds was stored at room temperature as directed by the sponsor. Setup of NR Assays. The NR Assays were performed as depicted in Figures 1 and 2. In brief, Step 1: A suspension of Reporter cells was prepared in cell recovery medium (CRM; containing 10\% charcoal stripped FBS). For antagonist assays, reporter cells were supplemented with a $2 \mathrm{x}$-EC80 concentration of the appropriate reference agonist. For agonist and antagonist assays, $100 \mathrm{ml}$ of the reporter cell suspension was dispensed into the wells of white, cell culture treated, 96-well assay plates. For agonist and antagonist assays, $2 \mathrm{x}$-concentration treatment media were prepared.

Step 2: Immediately prior to assay setup, test compounds were diluted using compound screening medium (CSM; containing 10\% charcoal stripped FBS) to generate ' $2 \mathrm{x}$-concentration' treatment media. $100 \mathrm{ml}$ of each treatment medium was dispensed into triplicate assay wells pre-dispensed with Reporter Cells. Assay plates were incubated at $37^{\circ} \mathrm{C}$ for $24 \mathrm{hr}$.

Step 3: Following the $24 \mathrm{hr}$ incubation period, treatment media were discarded and $100 \mu \mathrm{l} /$ well of Luciferase Detection Reagent was added. RLUs were quantified from each assay well to determine NR activity.

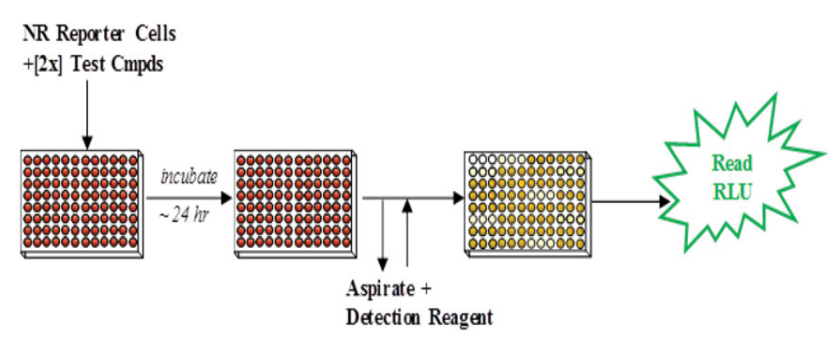

Figure 1: Agonist assays.

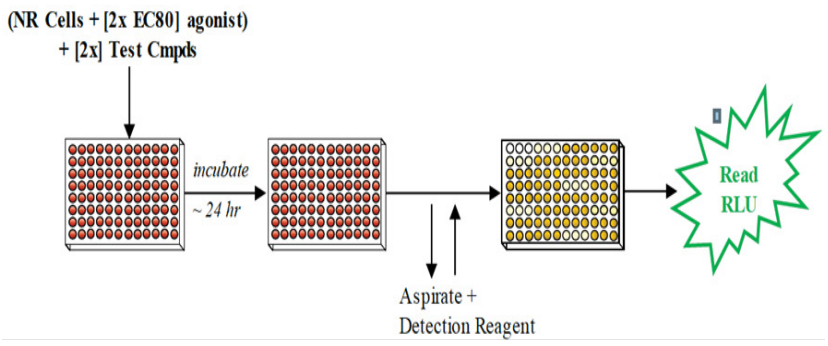

Figure 2: Antagonist assays. 


\section{Assay validation}

Reference compounds were utilized to confirm the performance of the specific lot of NR Reporter Cells treated with the Sponsor's test compounds. Reference compound and test compound assays were performed at the same time and, hence, were exposed to the same assay reagents and environmental conditions. Refer to individual data sets for the identities of specific reference agonist and antagonist and their respective treatment concentration ranges. Reference groups always include a 'Vehicle' control to determine background activity in the assay and to calculate fold-activation or percent-inhibition.

\section{Data reduction}

Microsoft Excel was used to manage and archive assay data, as well as to calculate average RLU values +/- Standard Deviation (SD), Foldactivation, Percent activation, Percent Coefficients of Variation (\%CV), and Z' values.

\section{Graphical data methods}

Dose-response curve (DRC) analyses of the reference compounds and test compounds were performed via non-linear curve-fitting of Fold-Activation vs. $\log$ (Compound) for agonist assays and percent inhibition vs. $\log$ (Compound) for antagonist and inverse agonist assays, using Graph Pad Prism software.

\section{Keap1-NRF2 assays}

All work was outsourced and performed by DiscoverX, Fremont, $\mathrm{CA}$, using their proprietary procedure. This is a target-specific, cellbased functional assay for NRF2 that provides a non-transcriptional response of activation-dependent NRF2 translocation to the nucleus.

\section{Assay measures}

Nuclear translocation of NRF2 following its disassociation from Keap1

Activation of the NRF2 response pathway

The control ligand was CDDO methyl ester.

\section{Experimental Procedure}

Compounds were tested in agonist and antagonist mode with the Keap1-NRF2 Biosensor Assay. For agonist assays, data was normalized to the maximal and minimal response observed in the presence of control ligand and vehicle. For antagonist assays, data was normalized to the maximal and minimal response observed in the presence of EC80 ligand and vehicle. An EC80 of $12 \mathrm{nM} \mathrm{CDDO}$ methyl ester was used.

\section{Cell handling}

1. PathHunter Pathway cell lines were expanded from freezer stocks according to standard procedures.

2. Cells were seeded in a total volume of $20 \mu \mathrm{L}$ into white walled, 384-well micro plates and incubated for the appropriate me prior to testing.

\section{Agonist format}

1. For agonist determination, cells were incubated with sample to induce response.

2. Intermediate dilution of sample stocks was performed to generate $5 \mathrm{X}$ sample in assay buffer.

3. $5 \mu \mathrm{L}$ of $5 \mathrm{X}$ sample was added to cells and incubated at $37^{\circ} \mathrm{C}$ or room temperature for 1.5-16 hours depending on the assay. Vehicle concentration was $1 \%$.

\section{Antagonist format}

1. For antagonist determination, cells were pre-incubated with antagonist followed by agonist challenge at the EC80 concentration.

2. Intermediate dilution of sample stocks was performed to generate $5 \mathrm{X}$ sample in assay buffer.

3. $5 \mu \mathrm{L}$ of $5 \mathrm{X}$ sample was added to cells and incubated at $37^{\circ} \mathrm{C}$ or room temperature for 60 minutes. Vehicle concentration was $1 \%$.

$4.5 \mu \mathrm{L}$ of $6 \mathrm{X}$ EC80 agonist in assay buffer was added to the cells and incubated at $37^{\circ} \mathrm{C}$ or room temperature for $1.5-16$ hours depending on the assay.

\section{Signal detection}

1. Assay signal was generated through a single addition of 12.5 or $15 \mu \mathrm{L}(50 \% \mathrm{v} / \mathrm{v})$ of PathHunter Detection reagent cocktail for agonist and antagonist assays respectively, followed by a one-hour incubation at room temperature. For some assays, activity was detected using a high sensitivity ti reagent (PathHunter FlashKit) to improve assay performance. In these assays, equal volume of detection reagent (25 or $30 \mathrm{uL}$ ) was added to the wells, followed by a one hour at room temperature.

2. Micro plates were read following signal generation with a PerkinElmer EnvisionTM instrument for chemiluminescent signal detection

\section{Data analysis}

1. Compound activity was analyzed using CBIS data analysis suite (ChemInnovation, CA).

2. For agonist mode assays, percentage activity was calculated using the following formula: \% Activity $=100 \% \mathrm{x}$ (mean RLU of test samplemean RLU of vehicle control) / (mean MAX RLU control ligand- mean RLU of vehicle control).

3. For antagonist mode assays, percentage inhibition was calculated using the following formula:

$\%$ Inhibition $=100 \% \times(1-$ (mean RLU of test sample-mean RLU of vehicle control)/(mean RLU of EC80 control- mean RLU of vehicle control)).

\section{Results and Discussion}

The AHR raw data results for agonist and antagonist are is shown in Tables 1 and 2, The agonist and antagonist graphs are shown in Figures 3-8. MeBio was used as a standard in the agonist assay and GNF-351 used as the standard in the antagonist assay. Keap1-NRF2 raw data are shown in Tables 3-5. From the Nuclear receptor agonist and antagonist assay Figures 5 and 6 it is clear that Metadichol is behaving like an inverse agonist. There is no known inverse agonist of AHR reported in literature.

In the Keap-NRF2 assay shown in Figure 7 it clear that Metadichol has no agonist activity but has an antagonist activity. But what is interesting is that the response is not $100 \%$ even at higher concentration and maximum response did not exceed $35 \%$.

Nutrition has a huge role in functioning of the human organism by affecting gene expression in specific tissues of the organism. This helps the organism to adapt to changes in the environment. The intracellular Nuclear receptors link diet and gene expression in response to small 
Citation: Raghavan PR (2017) Metadichol ${ }^{\circledR}$. A Novel Inverse Agonist of Aryl Hydrocarbon Receptor (AHR) and NRF2 Inhibitor. J Cancer Sci Ther 9: 661-668. doi:10.4172/1948-5956.1000489

\begin{tabular}{|c|c|c|c|c|c|c|c|c|c|c|}
\hline \multirow{2}{*}{$\begin{array}{c}\text { Compound } \\
\text { Water }\end{array}$} & \multicolumn{10}{|c|}{ Values } \\
\hline & $0.20 \%$ & 15,067 & 11,180 & 10,349 & 12,199 & 2,518 & 1.0 & 4.2 & 21 & - \\
\hline \multirow{7}{*}{ Metadichol $(\mu \mathrm{g} / \mathrm{ml})$} & 0.0073 & 10,986 & 8,085 & 10,559 & 9,877 & 1,566 & 0.81 & 3.4 & 16 & - \\
\hline & 0.024 & 9,304 & 7,012 & 5,776 & 7,364 & 1,790 & 0.60 & 2.5 & 24 & - \\
\hline & 0.081 & 8,291 & 6,874 & 7,178 & 7,448 & 746 & 0.61 & 2.5 & 10 & - \\
\hline & 0.27 & 7,445 & 7,537 & 6,870 & 7,284 & 361 & 0.60 & 2.5 & 5.0 & - \\
\hline & 0.90 & 3,513 & 5,732 & 5,247 & 4,830 & 1,167 & 0.40 & 1.6 & 24 & - \\
\hline & 3.0 & 3,056 & 2,112 & 2,966 & 2,712 & 521 & 0.22 & 0.92 & 19 & - \\
\hline & 10 & 302 & 262 & 286 & 283 & 20 & 0.023 & 0.10 & 7.1 & - \\
\hline \multirow{8}{*}{ Reference Antagonist: GNF351 (nM) } & 0.0038 & 84,571 & 80,274 & 96,520 & 87,121 & 8,418 & 7.1 & 30 & 9.7 & $Z^{\prime}$ \\
\hline & 0.19 & 110628 & 130,696 & 92,272 & 111,199 & 19,218 & 9.1 & 38 & 17 & - \\
\hline & 0.96 & 184984 & 156,008 & 155,234 & 165,409 & 16,957 & 14 & 56 & 10 & - \\
\hline & 4.8 & 229832 & 217,538 & 173,853 & 207,074 & 29,420 & 17 & 70 & 14 & 0.51 \\
\hline & 24 & 282781 & 262,276 & 269,296 & 271,451 & 10,421 & 22 & 92 & 3.8 & 0.85 \\
\hline & 120 & 238531 & 300,398 & 297,017 & 278,649 & 34,784 & 23 & 95 & 12 & 0.58 \\
\hline & 600 & 284983 & 326,358 & 270,364 & 293,902 & 29,043 & 24 & 100 & 10 & 0.66 \\
\hline & 3,000 & 266265 & 347,989 & 235,103 & 249,786 & 15,658 & 20 & 85 & 6.3 & 0.77 \\
\hline
\end{tabular}

Table 1: Agonist assay.

\begin{tabular}{|c|c|c|c|c|c|c|c|c|c|c|}
\hline \multirow{2}{*}{ Compound } & \multirow{2}{*}{ Conc. } & \multicolumn{3}{|c|}{ Human AhR antagonist assays } & \multirow{2}{*}{ AVG } & \multirow{2}{*}{ SD } & \multirow{2}{*}{ Fold-inhibition } & \multirow{2}{*}{$\%$ Inhibition } & \multirow{2}{*}{$\% \mathrm{CV}$} & \multirow{2}{*}{ Value } \\
\hline & & Luc1 & Luc2 & Luc3 & & & & & & \\
\hline Water & $0.20 \%$ & 231,269 & 274,275 & 272,890 & 259,478 & 24,440 & 1.0 & 0.0 & 9.4 & - \\
\hline \multirow{7}{*}{ Metadichol $(\mu \mathrm{g} / \mathrm{ml})$} & 0.0073 & 283,234 & 226,097 & 297,118 & 268,860 & 37,667 & 0.97 & -3.6 & 14 & - \\
\hline & 0.024 & 266,507 & 285,157 & 280,584 & 277,416 & 9,720 & 0.94 & -6.9 & 3.5 & - \\
\hline & 0.081 & 266,802 & 263,538 & 285,246 & 271,862 & 11,705 & 0.95 & -4.8 & 4.3 & - \\
\hline & 0.27 & 231,747 & 225,736 & 243,391 & 233,625 & 8,976 & 1.1 & 10 & 3.8 & - \\
\hline & 0.90 & 140,583 & 147,609 & 127,846 & 138,679 & 10,018 & 1.9 & 47 & 7.2 & - \\
\hline & 3.0 & 47,538 & 39,305 & 26,603 & 37,815 & 10,547 & 6.9 & 85 & 28 & - \\
\hline & 10 & 1,216 & 766 & 1,264 & 1,082 & 275 & 240 & 100 & 25 & - \\
\hline \multirow{8}{*}{$\begin{array}{l}\text { Reference Antagonist: } \\
\text { GNF351 (nM) }\end{array}$} & 0.61 & 267,661 & 254,185 & 214,007 & 245,284 & 27,912 & 1.1 & 5.5 & 11 & $Z^{\prime}$ \\
\hline & 2.4 & 235,944 & 271,376 & 260,974 & 256,098 & 18,212 & 1.0 & 1.3 & 7.1 & - \\
\hline & 9.8 & 269,755 & 247,412 & 204,923 & 240,697 & 32,934 & 1.1 & 7.2 & 14 & - \\
\hline & 39 & 241,635 & 231,420 & 226,931 & 233,329 & 7,536 & 1.1 & 10 & 3.2 & - \\
\hline & 156 & 175,211 & 154,885 & 188,760 & 172,952 & 17,050 & 1.5 & 33 & 10 & - \\
\hline & 625 & 67,127 & 70,861 & 94,960 & 77,649 & 15,107 & 3.3 & 70 & 19 & - \\
\hline & 2500 & 10,709 & 11,052 & 14,977 & 12,246 & 2,371 & 21 & 95 & 19 & 0.67 \\
\hline & 10000 & 202 & 128 & 218 & 183 & 48 & 1420 & 100 & 26 & 0.72 \\
\hline
\end{tabular}

Table 2: Antagonist assay.

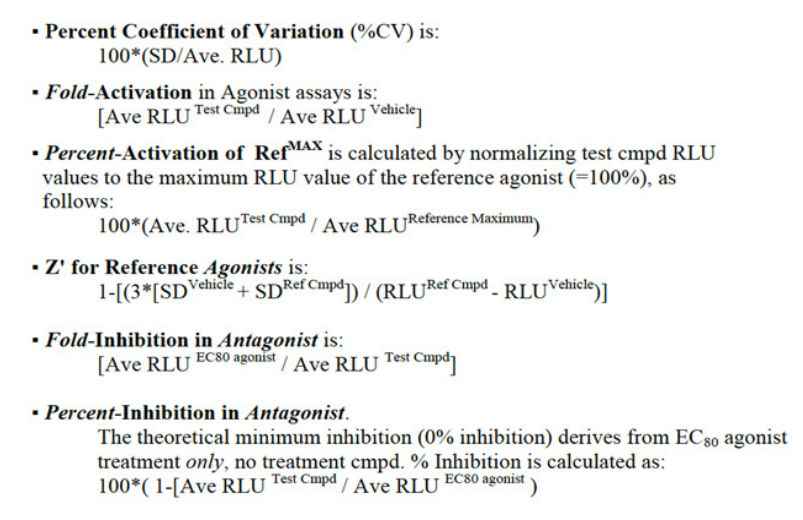

Figure 3: Microsoft Excel was used to manage and archive assay data.

lipophilic ligands derived from endocrine organs, metabolism, diet and the environment. Metadichol is composed of C-26, C-28, C-30 long chain alcohols and is an open chain analogue of 1,25 dihydroxy vitamin D3 the natural ligand of VDR which is a C27 alcohol. In addition, Metadichol is a ubiquitous lipid that is part of our diet [31] and is a lipophilic ligand. Most of the ligands for Nuclear receptors are lipids with the exception of the Thyroid receptor which has Iodine present. [32].

An inverse agonist binds to the same binding site on the receptor

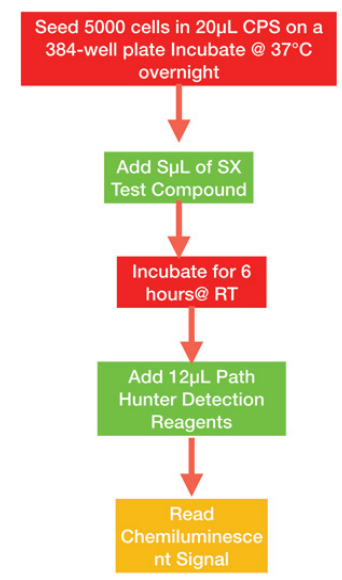

Figure 4: Assay protocol, The NRF2 response pathway. 
Citation: Raghavan PR (2017) Metadichol ${ }^{\circledR}$. A Novel Inverse Agonist of Aryl Hydrocarbon Receptor (AHR) and NRF2 Inhibitor. J Cancer Sci Ther 9: 661-668. doi:10.4172/1948-5956.1000489

as an agonist does but induces a biological response opposite to that of an agonist. A prerequisite for an inverse agonist response is that the receptor must have high constitutive or basal activity [33] in the absence of an identifiable ligand. An inverse agonist [34] can have effects

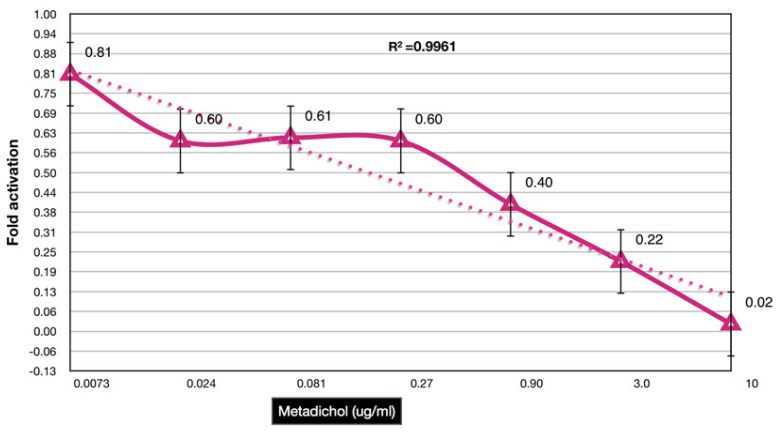

Figure 5: Agonist assay of Metadichol.

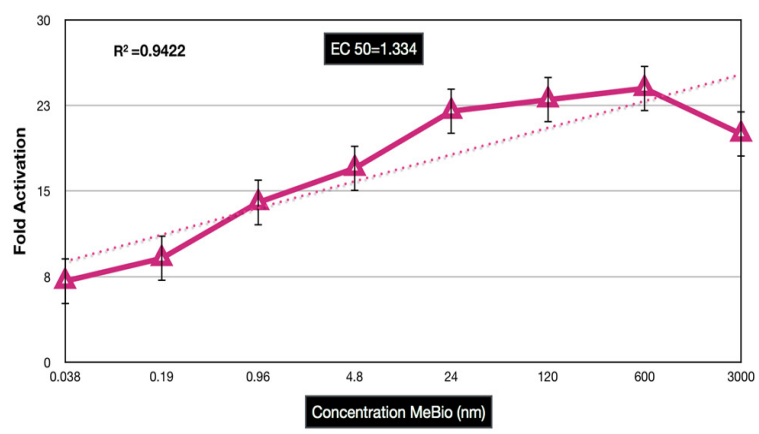

Figure 6: Standard agonist assay of MeBio.

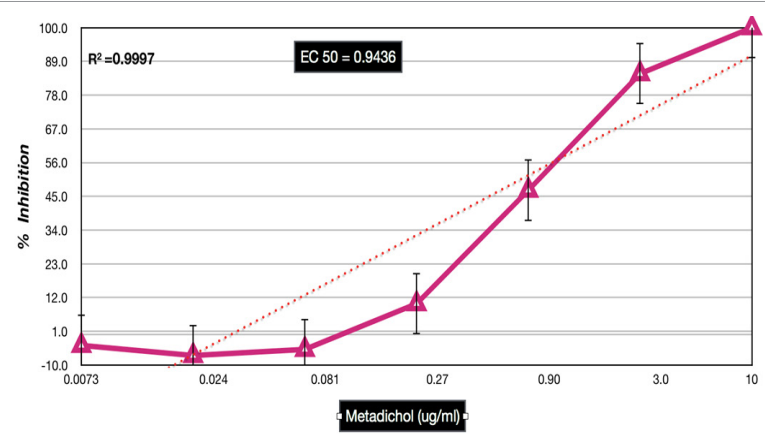

Figure 7: Antagonist assay of Metadichol.

\begin{tabular}{|c|c|c|c|}
\hline \multicolumn{4}{|c|}{ CDDO Methyl Ester } \\
\hline Well ID & Conc & Raw Value & Percent Efficacy \\
\hline B22 & $5.08 \mathrm{E}-06$ & 237800 & 2.0 \\
\hline B19 & $1.52 \mathrm{E}-05$ & 232800 & 1.4 \\
\hline B20 & $1.52 \mathrm{E}-05$ & 244000 & 2.8 \\
\hline B17 & $4.57 \mathrm{E}-05$ & 221200 & 0.1 \\
\hline B18 & $4.57 \mathrm{E}-05$ & 219000 & -0.2 \\
\hline B15 & 0.00013717 & 226400 & 0.7 \\
\hline B16 & 0.00013717 & 241400 & 2.5 \\
\hline B13 & 0.00041152 & 253400 & 3.9 \\
\hline B14 & 0.00041152 & 262800 & 5.0 \\
\hline B11 & 0.0012346 & 367200 & 17.2 \\
\hline B12 & 0.0012346 & 352200 & 15.5 \\
\hline
\end{tabular}

\begin{tabular}{|c|c|c|c|}
\hline B9 & 0.0037037 & 685600 & 54.6 \\
\hline B10 & 0.0037037 & 624400 & 47.4 \\
\hline B7 & 0.011111 & 927200 & 82.9 \\
\hline B8 & 0.011111 & 942400 & 84.7 \\
\hline B5 & 0.033333 & $1 \mathrm{E}+06$ & 94.2 \\
\hline B6 & 0.033333 & $1 \mathrm{E}+06$ & 105.8 \\
\hline B3 & 0.1 & $1 \mathrm{E}+06$ & 96.0 \\
\hline B4 & 0.1 & $1 \mathrm{E}+06$ & 110.4 \\
\hline
\end{tabular}

Table 3: Keap1-NRF2 raw data.

\begin{tabular}{|c|c|c|c|}
\hline Metadichol-Agonist assay & Conc & Raw value & Percent efficacy \\
\hline C21 & $9.77 \mathrm{E}-02$ & 273400 & 1.3 \\
\hline C22 & $9.77 \mathrm{E}-02$ & 275400 & 1.5 \\
\hline C19 & $1.95 \mathrm{E}-01$ & 254200 & -1.1 \\
\hline C20 & $1.95 \mathrm{E}-01$ & 281200 & 2.3 \\
\hline C18 & $3.91 \mathrm{E}-01$ & 257000 & -0.7 \\
\hline C15 & $3.91 \mathrm{E}-01$ & 248800 & -1.7 \\
\hline C16 & 0.78125 & 256200 & -0.8 \\
\hline C13 14 & 0.78125 & 267800 & 0.6 \\
\hline C11 & 1.5625 & 246800 & -2.0 \\
\hline C12 & 1.5625 & 244400 & -2.3 \\
\hline C9 10 & 3.125 & 255400 & -0.9 \\
\hline C7 & 3.125 & 255200 & -1.0 \\
\hline C8 & 6.25 & 268600 & 0.7 \\
\hline C5 & 6.25 & 279200 & 2.0 \\
\hline C6 & 12.5 & 296000 & 4.1 \\
\hline C3 & 12.5 & 277000 & 1.7 \\
\hline C4 & 25 & 269600 & 0.8 \\
\hline
\end{tabular}

Table 4: Metadichol-agonist assay Keap1-NRF2 raw data.

\begin{tabular}{|c|c|c|c|}
\hline Metadichol antagonist assay & Conc & Raw val & Percent efficacy \\
\hline $\mathrm{H} 21$ & $9.77 \mathrm{E}-02$ & 1195800 & -3.5 \\
\hline $\mathrm{H} 22$ & $9.77 \mathrm{E}-02$ & 1252000 & -9.8 \\
\hline H19 & 1.95E-01 & 1239600 & -8.4 \\
\hline $\mathrm{H} 2 \mathrm{O}$ & 1.95E-01 & 1124000 & 4.5 \\
\hline $\mathrm{H} 17$ & $3.91 \mathrm{E}-01$ & 1125000 & 4.4 \\
\hline $\mathrm{H} 18$ & $3.91 \mathrm{E}-01$ & 1125400 & 4.3 \\
\hline $\mathrm{H} 16$ & 0.78125 & 1050000 & 12.8 \\
\hline $\mathrm{H} 16$ & 0.78125 & 1116800 & 5.3 \\
\hline $\mathrm{H} 13$ & 1.5625 & 1001000 & 18.2 \\
\hline $\mathrm{H} 14$ & 1.5625 & 1072800 & 10.2 \\
\hline $\mathrm{H} 11$ & 3.125 & 977800 & 20.8 \\
\hline $\mathrm{H} 12$ & 3.125 & 913000 & 28.1 \\
\hline H9 & 6.25 & 984400 & 20.1 \\
\hline $\mathrm{H} 10$ & 6.25 & 986800 & 19.8 \\
\hline $\mathrm{H} 7$ & 12.5 & 871000 & 32.8 \\
\hline $\mathrm{H} 8$ & 12.5 & 914600 & 27.9 \\
\hline H5 & 25 & 886000 & 31.1 \\
\hline $\mathrm{H} 6$ & 25 & 879600 & 31.8 \\
\hline $\mathrm{H} 3$ & 50 & 853800 & 34.7 \\
\hline $\mathrm{H} 4$ & 50 & 831400 & 37.2 \\
\hline
\end{tabular}

Table 5: Metadichol-antagonist assay Keap1-NRF2 raw data.

similar to that of an antagonist but cause a distinct set of downstream biological responses. They block the effects of binding agonists like a classical antagonist but also inhibit the basal activity of the receptor. 
Antihistamines were classified previously as antagonists of histamine $\mathrm{H} 1$ receptors have been reclassified as inverse agonists [35]. AHR is constitutively active in prostate cancer [36,37]. Tumors and tumor cell lines have elevated AHR levels. AHR is chronically activated in tumors [38]. Masafumi et al. [39] created a transgenic mouse lines expressing the constitutive form of the AHR in keratinocytes. In these lines of mice, and showed that the AHR activity was constitutively enhanced in the absence of ligands, and direct effects of PAHs (poly aromatic hydrocarbons) and their metabolites could be ignored.

The enhanced level of expression of AHR in human tumors is the ability of NFkB to increase AHR expression, which correlates with inflammatory status [40]. There is a need for high-affinity AHR ligands that exhibit improved biological profiles Metadichol, as we have shown,

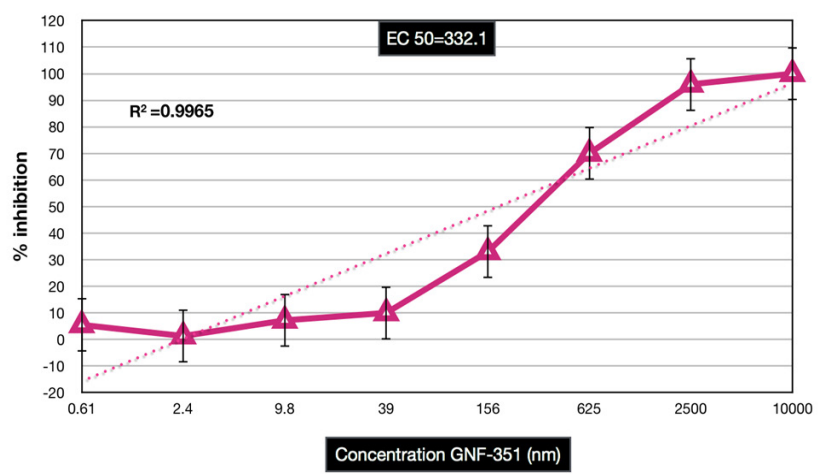

Figure 8: Antagonist assay of standard GNF-351.
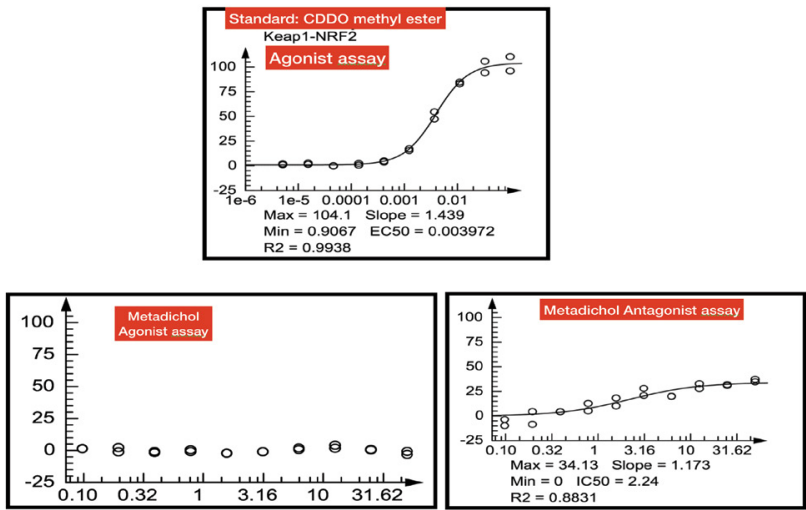

Figure 9: Keap-NRF2 assay.

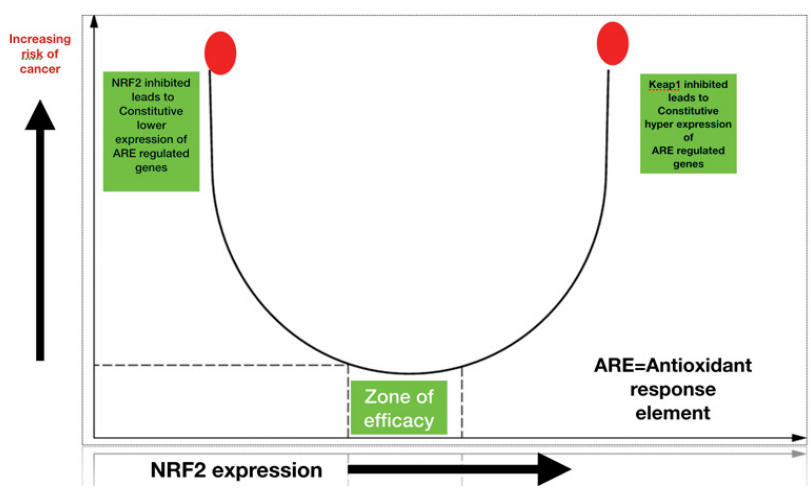

Figure 10: Metadichol dose response is probably U-shaped.

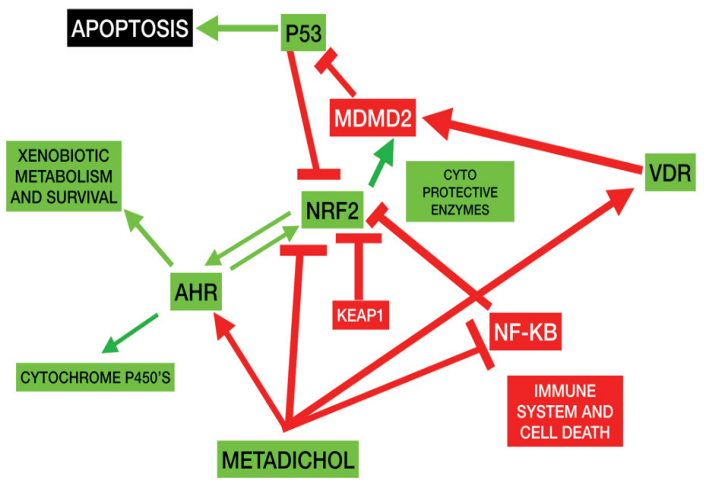

Figure 11: Possible signaling pathways by the binding actions of Metadichol on AHR and NRF2.

is an inhibitor of NF-KB. The emerging role of the AHR in immune tolerance, and clinical testing of AHR antagonists like Metadichol as an adjunct to immunotherapy is an approach that needs to be pursued. We have already shown that Metadichol is an inverse agonist of VDR. No known inverse agonist of AHR and VDR are known. What has been postulated is that an inverse agonist will have effects opposite to that of an agonist. Inverse agonists not only block constitutive responses of receptors but also to activate and regulate seven-transmembrane receptor (GPCR) signaling [41]. Our studies in human subjects suggest that Metadichol behaves more like a protean agonist $[42,43]$. For example, we have shown that it lowers insulin levels as well as increases Insulin levels. We believe that it also acts as a protean agonist i.e. it acts as an inverse agonist where there is constitutive activity and as an agonist when there is none.

\section{NRF2 and Metadichol}

In the Keap-NRF2 assay shown in Figure 9 it clear that Metadichol has no agonist activity but has an antagonist activity. But what is interesting is that the response is not $100 \%$ even at higher concentration, and maximum response does not exceed 35\%. The barrier to be overcome in cancer treatment is the resistance of cancer cells to chemotherapy. High constitutive expression of Nrf2 is found in many types of cancers, that leads to an environment conducive to cancer cell survival. Thus, there is a need for optimal inhibition of NRF2 expression to overcome the resistance of cancer cells to chemotherapy. Metadichol dose response is probably U-shaped (Figure 10). U-shaped. Such shaped curves are seen with some chemo preventive agents, such as vitamin $\mathrm{D}$ and selenium as well as for some chemotherapeutic drugs. Metadichol does not activate NRF2 and thus could be useful to increase the effectiveness of cancer treatment. [44]. The NRF2 pathway is the useful target in the treatment of diabetes [45]. We have published a case study where Metadichol normalized blood sugar levels [46].

\section{Conclusion}

The results that we have obtained with Metadichol that it is an inverse agonist of AHR show that possible signaling pathways by the binding actions of Metadichol on AHR and NRF2 is shown in Figure 11. Thus, Metadichol could be useful in cancers where there are increased levels of AHR expressed. The discovery of functional cooperation between AHR and Nrf2 has significant biological implications [47,48]. AHR has been associated with carcinogenesis, but Nrf2 is associated with cytoprotection against degenerative diseases. Cross-talk between these two transcription factors can be exploited by Metadichol to therapeutic advantage and improve outcomes in cancer chemotherapy 
Citation: Raghavan PR (2017) Metadichol ${ }^{\circledR}$. A Novel Inverse Agonist of Aryl Hydrocarbon Receptor (AHR) and NRF2 Inhibitor. J Cancer Sci Ther 9: 661-668. doi:10.4172/1948-5956.1000489

where there is a need to control AHR and NRF2 level. Metadichol by binding to VDR could be useful given the role of Vitamin D in cancer therapy [49]. Metadichol by leveraging targets AHR, VDR, NRF2 that are synergistic can produce greater levels of efficacy and synergy combinations and positively impacts the complex systems biology of human disease. Being not toxic [50-52] and is a huge advantage over all drugs in development and allows for clinical testing in humans.

\section{References}

1. Raghavan PR (2017) Rheumatoid arthritis and osteoporosis: A case study. J Arthritis 6: 240 .

2. Barouki R, Coumoul X, Fernandez-Salguero PM (2015) The aryl hydrocarbon receptor, more than a xenobiotic-interacting protein. FEBS Lett 581: 36083615.

3. Noakes R (2015) The aryl hydrocarbon receptor: A review of its role in the physiology and pathology of the integument and its relationship to the tryptophan metabolism. Int J Tryptophan Res 8: 7-18.

4. Murray IA, Flaveny CA, DiNatale BC, Chairo CR, Schroeder JC, et al. (2010) Antagonism of aryl hydrocarbon receptor signalling by 6,2',4'-trimethoxyflavone. J Pharmacol Exp Ther 332: 135-144.

5. Francisco $\mathrm{J}(2008)$ Control of $t(\mathrm{reg})$ and $\mathrm{T}(\mathrm{H}) 17$ cell differentiation by the aryl hydrocarbon receptor. Nature 453: 65-71.

6. Eun-Young Choi, Lee H (2012) Development of novel CH223191-based antagonists of the aryl hydrocarbon receptor. Mol Pharmacol 81: 3-11.

7. Boitano AE, Wang J, Romeo R, Parer AE, Sutton SE, et al. (2010) Aryl hydrocarbon receptor antagonists promote the expansion of human hematopoietic stem cells. Science 10: 1345-1348.

8. Korn T (2010) How T cells take developmental decisions by using the ary hydrocarbon receptor to sense the environment. PNAS 107: 20597-20598.

9. Koliopanos A, Kleeff J, Xiao Y, Safe S, Zimmermann A, et al. (2002) Increased aryl hydrocarbon receptor expression offers a potential therapeutic target for pancreatic cancer. Oncogene 21: 6059-6070.

10. Gluschnaider U, Hidas G, Cojocaru G, Yutkin V, Ben-Neriah Y, et al. (2010) Beta-TCP inhibition reduces prostate cancer cell growth via upregulation of the aryl hydrocarbon receptor. PLoS One 5: e9060.

11. Ishida M, Mikami S, Kikuchi E, Kosaka T, Miyajima A, et al. (2010) Activation of the aryl hydrocarbon receptor pathway enhances cancer cell invasion by upregulating the MMP expression and is associated with poor prognosis in upper urinary tract urothelial cancer. Carcinogenesis 31: 287-295.

12. Portal-Nuñez S, Shankavaram UT, Rao M, Datrice N, Atay S, et al. (2012) Aryl hydrocarbon receptor-induced adrenomedullin mediates cigarette smoke carcinogenicity in humans and mice. Cancer Res 72: 5790-5800.

13. Zhang J1, Zong H, Li S, Zhang D, Zhang L, et al. (2012) Activation of aryl hydrocarbon receptor suppresses invasion of esophageal squamous cell carcinoma cell lines. Tumor 98: 152-157.

14. Jaffrain-Rea ML, Angelini M, Gargano D, Tichomirowa MA, Daly AF, et al. (2009) Expression of aryl hydrocarbon receptor (AHR) and AHR-interacting protein in pituitary adenomas: Pathological and clinical implications. Endocr Relat Cancer 16: 1029-1043.

15. Gramatzki D, Pantazis G, Schittenhelm J, Tabatabai G, Köhle C, et al. (2009) Aryl hydrocarbon receptor inhibition downregulates the TGF-beta/Smad pathway in human glioblastoma cells. Oncogene 28: 2593-2605.

16. Stanford EA, Wang Z, Novikov O, Mulas F, Landesman-Bollag E, et al. (2016) The role of the aryl hydrocarbon receptor in the development of cells with the molecular and functional characteristics of cancer stem-like cells. BMC Biol 16: $14-20$.

17. Nguyen T, Nioi P, Pickett CB (2009) The Nrf2-antioxidant response element signaling pathway and its activation by oxidative stress. J Biol Chem 284 : 13291-5.

18. Lü JM, Lin PH, Yao Q, Chen C (2010) Chemical and molecular mechanisms of antioxidants: Experimental approaches and model systems. J Cell Mol Med 14: 840-60.

19. Cook AL, Vitale AM, Ravishankar S, Matigian N, Sutherland GT, et al. (2011)
NRF2 activation restores disease-related metabolic deficiencies in olfactory neurosphere-derived cells from patients with sporadic Parkinson's disease. PLoS One 6: e21907

20. Olagnier D, Lavergne RA, Meunier E, Lefèvre L, Dardenne C, et al. (2011) Nrf2, a PPAR-gamma alternative pathway to promote CD36 expression on inflammatory macrophages: implication for malaria. PLoS Pathog 7: e1002254

21. Harvey CJ, Thimmulappa RK, Sethi S, Kong X, Yarmus L, et al. (2011) Targeting Nrf2 signaling improves bacterial clearance by alveolar macrophages in patients with COPD and a mouse model. Sci Trans Med 3: 78.

22. Malhotra D, Thimmulappa RK, Mercado N, Ito K, Kombairaju P, et al. (2011) Denitrosylation of HDAC2 by targeting Nrf2 restores gluco- cortico-steroid sensitivity in macrophages from COPD patients. J Clin Invest 121: 4289-302.

23. Linker RA, Lee DH, Ryan S, van Dam AM, Conrad R, et al. (2011) Fumaric acid esters exert neuroprotective effects in neuroinflammation via activation of the Nrf2 antioxidant pathway. Brain 134: 678-692.

24. Ren D, Villeneuve NF, Jiang T, Wu T, Lau A, et al. (2011) Brusatol enhances the efficacy of chemotherapy by inhibiting the Nrf2-mediated defense mechanism. Proc Natl Acad Sci USA 108: 1433-1438.

25. Cho HY, Kwak MK, Pi J (2013) Nrf2 in host defense: Over the rainbow. Oxid Med Cell Longev 975839.

26. Cho HY, Kleeberger SR (2010) Nrf2 protects against airway disorders. Toxicol Appl Pharmacol. 244: 43-56.

27. Xue P, Hou Y, Chen Y, Yang B, Fu J, et al. (2013) Adipose deficiency of Nrf2 in ob/ob mice results in severe metabolic syndrome. Diabetes 62: 845-854

28. Miao W, Hu L, Scrivens PJ, Batist G (2005) Transcriptional regulation of NF E2 p45-related factor (NRF2) expression by the aryl hydrocarbon receptorxenobiotic response element signaling pathway: Direct cross-talk between phase I and II drug-metabolizing enzymes. J Biol Chem 280: 20340-20348.

29. Shin S, Wakabayashi N, Misra V, Biswal S, Lee GH (2007) NRF2 modulates aryl hydrocarbon receptor signaling: Influence on adipogenesis. Mol Cell Biol 27: 7188-7197.

30. Noda S, Harada N, Hida A, Fujii-Kuriyama Y, Motohashi H, et.al, (2003) Gene expression of detoxifying enzymes in AHR and Nrf2 compound null mutant mouse. Biochem Biophys Res Commun 303: 105-111.

31. Hargrove JL, Greenspan P, Hartle DK (2004) Nutritional significance and metabolism of very long chain fatty alcohols and acids. Experiment Biol Medicine 229: 215-226.

32. Evans RM, Mangelsdorf DJ (2014) Nuclear Receptors, RXR and the Big Bang Cell 157: 255-266.

33. Kenakin T (2004) Efficacy as a vector: The relative prevalence and paucity of inverse agonism. Molecular Pharmacol 65: 2-11.

34. Greasley PJ, Clapham JC (2006) Inverse agonism or neutral antagonism a G-protein coupled receptors: A medicinal chemistry challenge worth pursuing? Eur J Pharmacol 553: 1-9.

35. Leurs R, Church MK, Taglialatela M (2002) H1-Antihistamines: Inverse agonism anti-inflammatory actions, and cardiac effects. Clin Exp Allergy 32: 489-498.

36. Richmond O, Ghotbaddini M, Allen C, Walker A, Zahir S, et al. (2014) The ary hydrocarbon receptor is constitutively active in advanced prostate cancer cells. PLoS ONE 9: e95058.

37. Ching Yi C (1998) Constitutive activation of the aromatic hydrocarbon receptor Mol Cell Biol 18: 525-535.

38. Murray IA, Patterson AD, Perdew GH (2014) AH receptor ligands. In Cancer: Friend of foe. Nat Rev Cancer 14: 801-814.

39. Tauchiet M (2012) Constitutive expression of aryl hydrocarbon receptor in keratinocytes causes inflammatory skin lesions. Mol Cell Biol 32: 1759.

40. Vogel CF, Khan EM, Leung PS, Gershwin ME, Chang WL, et al. (2014) Crosstalk between aryl hydrocarbon receptor and the inflammatory response: A role for nuclear factor-kappa B. J Biol Chem 289: 1866-1875.

41. Dupré DJ, Rola-Pleszczynski M, Stanková J (2004) Inverse agonism: More than reverting constitutively active receptor signaling. Biochem Cell Biol 82 $676-680$.

42. Kenakin $T$ (2007) Functional selectivity through protean and biased agonism Who steers the ship? Mol Pharmacol 72: 1393-1402. 
Citation: Raghavan PR (2017) Metadichol ${ }^{\circledR}$. A Novel Inverse Agonist of Aryl Hydrocarbon Receptor (AHR) and NRF2 Inhibitor. J Cancer Sci Ther 9: 661-668. doi:10.4172/1948-5956.1000489

43. Neubig RR (2007) Missing links, mechanisms of protean agonism. Mol Pharmacol 171: 200-1202.

44. Waters DJ, Chiang EC, Bostwick DG (2008) The art of casting nets: fishing for the prize of personalized cancer prevention. Nutr Cancer 60: 1-6.

45. Dieter BP (2014) Dysregulation of Nrf2 signaling in diabetes: An Opportunity for a multi-target approach. J Diabetes Metab 6: 475.

46. Raghavan PR (2106) Metadichol and type 2 diabetes A case report. J Sci Healing Outcomes 8: 5-10.

47. Wakabayashi N, Slocum SL, Skoko JJ, Shin S, Kensler TW, et al. (2010) When NRF2 Talks, Who's Listening? Antioxid Redox Signal 13: 1649-1663.

48. Vousden KH, Ryan KM (2009) P53 and metabolism. Nat Rev Cancer 9: 691-700.
49. Feldman D, Krishnan AV, Swami S, Giovannucci E, Feldman BJ (2014) The role of vitamin $D$ in reducing cancer risk and progression. Nat Rev Cancer 14: 342-357.

50. Alemán CL, Más R, Hernández C, Rodeiro I, Cerejido E, et al. (1994) A 12-month study of policosanol oral toxicity in Sprague Dawley rats. Toxicol Lett 70: 77-87.

51. Alemán CL, Más Ferreiro R, Noa Puig M, Rodeiro Guerra I, Hernández Ortega C (1994) Carcinogenicity of policosanol in Sprague-Dawley rats: A 24-month study. Teratog Carcinog Mutagen 14: 239-249.

52. Alemán CL, Puig MN, Elías EC, Ortega CH, Guerra IR, et al. (1995) Carcinogenicity of policosanol in mice: An 18-month study. Food Chem Toxicol 33: $573-378$ 